\section{Serotonin syndrome caused by a moclobemide-clomipramine interaction}

Drs Olav Spigset and Tom MjörNDAL (Division of Clinical Pharmacology, Umeå University Hospital, Sweden) and Dr Olof Lövheim (Department of Internal Medicine, Örnsköldsvik Hospital, Sweden) write: The serotonin syndrome is a potentially lethal, toxic hyperserotoninergic state, most commonly resulting from an interaction between irreversible, non-selective monoamine oxidase inhibitors and agents increasing central nervous serotoninergic activity, such as serotonin reuptake inhibitors or tryptophan To our knowledge, the combination of reversible monoamine oxidase inhibitors and serotoninergic agents has never been reported to cause the serotonin syndrome. We present here a case of serotonin syndrome induced by the reversible monoamine oxidase A inhibitor moclobemide and the serotonin reuptake inhibitor clomipramine.

A 76 year old woman with depression and Parkinson's disease had been treated with antidepressants for several years, the last months with clomipramine $50 \mathrm{mg} /$ day. Other medications were levodopa-benserazide, bromocriptine, triazolam, diflunisal, dextropropoxyphene, oestriol, and lactulose. On the day before admission clomipramine had been changed to moclobemide $300 \mathrm{mg} /$ day. Next day the patient presented at the hospital somnolent, confused, and feverish (temperature $39 \cdot 0^{\circ} \mathrm{C}$ ). All tests for infectious diseases gave negative results. Serum creatine kinase activity was, however, $35.1 \mu \mathrm{kat} / \mathrm{l}$ (normal $<2.5$ $\mu \mathrm{kat} / \mathrm{l}$ ), indicating a muscular disorder. Her regular medication was discontinued. The next day her temperature rose to $40.9^{\circ} \mathrm{C}$, and her general condition and mental status were further impaired. She developed increased muscle stiffness and showed widespread myoclonus and intermittent convulsive attacks. One day later her general condition started to improve, and all ordinary drugs, except antidepressants, were restarted. After a few days she had recovered fully.

Our patient fulfilled the diagnostic criteria for the serotonin syndrome suggested by Sternbach.' Clomipramine has a half life varying between 22 and 84 hours, ${ }^{2}$ indicating that slow metabolisers could retain pharmacologically active amounts of the drug for one to two weeks after withdrawal of the drug. Neuroleptic malignant syndrome, described after withdrawal of levodopa and bromocriptine, ${ }^{3}$ seems unlikely as the symptoms were evident before these drugs were discontinued. Interactions between irreversible, nonselective monoamine oxidase inhibi- tors and levodopa and dextropropoxyphene have been reported,' but the symptoms were totally different from those in our patient.

Concomitant treatment with moclobemide and selective serotonin reuptake inhibitors should be used with great care and reserved for special cases. Serotonin reuptake inhibitors should have been discontinued for some time (depending on the half life of the drug and its metabolites) before treatment with moclobemide is started.

1 Stembach $\mathrm{H}$. The serotonin syndrome. $A m$ Psychiatry 1991;148:705-13.

2 Balant-Gorgia AE, Gex-Fabry M, Balant LP. Clinical pharmacokinetics of clomipramine. Clin Pharmacokinet 1991;20:447-62.

3 Rainer C, Scheinost NA, Lefeber EJ. Neuroleptic malignant syndrome. When levodopa withdrawal is the case. Postgrad Med 1991, 89:175-8.

4 Stockley IH. Drug interactions. 2nd ed. Oxford: Blackwell Scientific, 1991

\section{Hepatitis associated with terbinafine treatment}

Drs G Lowe, C Green, and P Jennings (Ninewells Hospital, Dundee DD1 9SY) write: Terbinafine is a new systemic fungicidal medication which is well tolerated with few side effects.' We report a case of acute hepatitis which occurred during treatment with this drug.

A 52 year old white man started taking terbinafine $250 \mathrm{mg}$ daily for a Trichophyton rubrum skin infection. After eight days he ran out of pills; treatment was restarted after six days. Four days later he developed lassitude, upper abdominal pain, biliary regurgitation, nausea, and dark urine. He immediately stopped treatment but did not seek medical advice until eight days later, at which time there was mild hepatic tenderness and raised concentration of alanine transaminase (470 U/l), $\gamma$-glutamyltransferase (143 U/l), alkaline phosphatase (193U/1), and bilirubin $(29 \mu \mathrm{mol} / \mathrm{l})$. Routine haematology and an autoantibody screen gave negative results. Serial investigations for hepatitis A, B and $C$ viruses, cytomegalovirus, and Epstein-Barr virus showed no evidence of current infection. $\mathrm{Ab}$ dominal ultrasound showed norma liver, gallbladder, and bile duct with no gallstones. He had no history of liver disease, of recent vaccination, or of blood transfusion or anaesthesia; alcohol intake was minimal; and he was taking no other medication Alanine transaminase values peaked 10 days later ( 35 days after starting and 18 days after discontinuing treatment) at $930 \mathrm{U} / \mathrm{l}$ but his symptoms resolved within one month and his liver function values returned to normal within two months.

Up to September 1992 seven cases of hepatobiliary disorders of varying pattern and severity associated with terbinafine had been reported to the Committee on Safety of Medicines (personal communication); total worldwide exposure to terbinafine amounts to about 95000 patients (Sandoz Pharmaceuticals, personal communication). Although we cannot completely rule out an alternative cause for our patient's hepatitis, the evidence suggests an idiosyncratic reaction to terbinafine. The interruption in treatment might be important, suggesting an initial period of sensitisation followed by accelerated hypersensitivity.

\section{Villars V, Jones TC. Present status of the efficacy and tolerability of terbinafine (Lamisil) used systemically in the treatment} Dermatol Treat 1990;1(suppl 2):33-8.

\section{Vaccine related glomerulonephritis}

Drs S Y TAN and A D Cumming (Medical Renal Unit, Royal Infirmary, Edinburgh EH3 9YW) write: We report a case of crescentic glomerulonephritis due to antiglomerular basement membrane disease after pneumococcal vaccination.

A previously healthy 30 year old man required emergency splenectomy in 1980 after a road accident. He remained well with no treatment and no antibiotic prophylaxis until December 1990, when he was admitted with pneumococcal septicaemia and acute renal failure. Treatment was complicated by penicillin allergy. $\mathrm{He}$ improved with eythromycin, and his renal function recovered without the need for dialysis. He remained stable until six months later, when he was given pneumococcal vaccine (Pneumovax) as further protection against pneumococcal sepsis. His medication at that time consisted of nifedipine retard, bendrofluazide, cimetidine, and erythromycin as prophylaxis. Two weeks after his vaccination he became unwell and was readmitted with oliguric acute renal failure.

Renal biopsy showed crescentic glomerulonephritis with linear staining of glomerular basement membrane on immunofluorescence. Anti-glomerular basement membrane titre was $119 \%$ (normal $<13 \%$ ) and antineutrophil cytolasmic antibody titre $6 \%$ (normal $<16 \%$ ). Treatment was started with plasma exchange, cyclophosphamide, methylprednisolone, and haemodialysis. He recovered renal function; on discharge plasma creatinine concentration was $160 \mu \mathrm{mol} / \mathrm{l}$ and anti-glomerular basement membrane titre $25 \%$. Retrospective analysis of blood samples taken six months earlier, during his septic illness, showed normal anti-

glomerular basement membrane and antineutrophil cytolasmic antibody titres.

None of his oral medication has been implicated as a cause of antiglomerular basement membrane disease, although erythromycin has been reported to cause interstitia nephritis' and was therefore stopped. $\mathrm{He}$ continued to take his othe medication without any complications.

Glomerulonephritis has been reported after vaccination. 2.5 $^{-5}$ In these cases it was suggested that components of the vaccine might have acted as an adjuvant to stimulate antibody production to exogenous or endogenous antigen, leading to glomerulonephritis. With multiple repeat vaccinations, as in the case reported by Boulton-Jones et al, antibody production and glomerulonephritis persisted despite treatment with immunosuppression However, the glomerulonephritis resolved spontaneously, with satisfactory recovery of renal function, once self administration of vaccine was stopped. In our patient the pneumococcal vaccine might have acted as an adjuvant to produce the sequence of events observed, and antibody production was not sustained by further exposure to vaccine adjuvant. Revaccination with Pneumovax is not recommended (Thomas Morson Pharmaceuticals) and our patient had had severe pneumococcal sepsis only six months before vaccination.

Glomerulonephritis after pneumococcal vaccine has not previously been described, and despite the common use of vaccines from infancy onwards vaccine related glomerulonephritis remains uncommon..$^{\circ}$ In our patient the threshold for the potential adjuvant effects of pneumococcal vaccine might have been lowered by an immune system primed by recent severe pneumococcal sepsis. The role of pneumococca vaccination should be considered in patients with a history of pneumococcal sepsis, and perhaps pneumococcal antibody titres should be measured before vaccination is contemplated.

Rosenfeld J, Gura V, Boner G, Ben-Bassat $M$ Livni $S$. Interstitial nephritis with acute renal failure after erythromycin. $B M Y$ I 1983 286:938-9.

2 Bishop WB, Carlton RF, Sanders LL. Diffuse vasculitis and death after hyperimmuniza- 